\title{
PREVISÃO DO NÚMERO DE DIAS DE CHUVA PARA A METADE SUL DO RIO GRANDE DO SUL UTILIZANDO A TEMPERATURA DA SUPERFÍCIE DO MAR (TSM)
}

\author{
GRACIELA REDIES FISCHER ${ }^{1}$, GILBERTO BARBOSA DINIZ ${ }^{2}$ E JULIO RENATO QUEVEDO \\ MARQUES $^{2}$
}
${ }^{1}$ Universidade Federal de Pelotas (UFPel), Faculdade de Meteorologia, Departamento de Meteorologia, Campus Universitário UFPel, Caixa Postal 354, Pelotas, RS, Brasil, CEP: 96010-900.
E-mail: graciela_fischer@yahoo.com.br
${ }^{2}$ Universidade Federal de Pelotas (UFPel), Faculdade de Meteorologia, Centro de Pesquisas e Previsão Meteorológicas/ UFPel. Av. Ildefonso Simões Lopes, 2751. Pelotas, RS, Brasil, CEP: 96060-290. E-mail: gilberto@ufpel.edu.br, jmarque_fmet@ufpel.edu.br

Recebido Março 2007 - Aceito Novembro 2007

\begin{abstract}
RESUMO
Este trabalho apresenta um modelo de previsão climatológica para o Número de Dias de Chuva (NDC) de algumas estações meteorológicas do Rio Grande do Sul, utilizando as Temperaturas da Superfície do Mar (TSM) como variáveis preditoras. Nesta pesquisa foram usados dois conjuntos de dados: o primeiro formado por dados mensais de NDC de 5 estações meteorológicas da metade sul do Estado, para o período de 1982 a 2005; o segundo, composto por dados de TSM, para o período de 1982 a 2005. A série foi dividida em dois períodos: o dependente, compreendendo o intervalo de 1982 a 2002, para determinação das equações preditivas, bem como os coeficientes de regressão, e o período independente, cujo intervalo foi de 2003 a 2005, para validação do modelo. Os dados de TSM foram utilizados para, através das equações de regressão, estabelecer as relações entre as variáveis. Para as duas regiões e para todos os meses analisados, obtiveram-se bons resultados na previsão de NDC. A série de dados prevista e a observada seguem um mesmo padrão de distribuição desta variável, embora existam alguns valores previstos que apresentam diferenças dos observados, essas não são significativas. No período independente, a série prevista mostra as maiores diferenças em relação aos valores observados.
\end{abstract}

Palavras-chave: Previsão Climática, Número de Dias de Chuva, Temperatura da Superfície do Mar, Rio Grande do Sul.

ABSTRACT: NUMBER OF RAINFALL DAYS FORECASTING FOR THE SOUTHERN HALF OF RIO GRANDE DO SUL USING THE SEA SURFACE TEMPERATURE (SST)

This study presents a climate forecasting model of the Number of Rainfall Days (NRD) for some meteorological stations in Rio Grande do Sul using the Sea Surface Temperatures (SST) as the predictable variables. Two sets of data were used in this research: the monthly data of NRD, which were obtained from 5 meteorological stations located in the southern half of Rio Grande do Sul, in the period of 1982 until 2005; and the SST data, measured in the same period. This series was divided in two periods: the dependent period is from 1982 to 2002, and it was used to determine the predictable equations and the regression coefficients; and the independent period, which is from 2003 to 2005 , and was used to validate the model. The SST data were employed to establish the relations between the variables through the regression analysis. Good results were obtained in the prediction of the NRD for the regions and all the months analyzed. The predictable and observed data had a very similar distribution of the variables. Although there was some predictable values that differed from the observed ones, but these differences were not significant. The higher differences between the foreseen and the observed values occurred in the independent period.

Keywords: Climate Forecasting, Number of Rainfall Days, Sea Surface Temperature, Rio Grande do Sul. 


\section{INTRODUÇÃO}

O Estado do Rio Grande do Sul, por suas características geográficas e climatológicas, em função não só da latitude e da longitude, mas também pela entrada dos sistemas meteorológicos, como frentes e massas de ar, carece de estudos mais apropriados sobre determinadas variáveis meteorológicas que interferem na produção agropecuária e na qualidade da vida humana.

A precipitação pluvial medida em estações meteorológicas nem sempre é um bom parâmetro para avaliar se a disponibilidade hídrica em determinado período é suficiente para o atendimento das necessidades das culturas agrícolas. Os totais de precipitação pluvial correspondem a todo o período considerado, não sendo levado em conta se foram bem distribuídos ao longo do período ou se ocorreram em um único dia.

Com o Número de Dias de Chuva (NDC), é possível ter-se uma idéia da intensidade da precipitação pluvial, pois ao se analisar o mesmo total de chuva em intervalos de tempo distintos, revela-se qual a intensidade e a variabilidade quantitativa e qualitativa da mesma.

Segundo Fontana (2002) o NDC aumenta de oeste para leste no Estado do Rio Grande do Sul, atingindo os maiores valores na Região Ecoclimática do Planalto Superior e Serra do Nordeste (Região Ecoclimática 3). Este padrão de NDC é consistente tanto para o total anual como para as quatro estações do ano.

A precipitação pluvial anual média do Rio Grande do Sul é da ordem de $1.540 \mathrm{~mm}$, sendo superior a $1.500 \mathrm{~mm}$ na metade norte do Estado e inferior a este valor na metade sul (BERLATO, 1992). Sua distribuição nas quatro estações do ano é bastante uniforme, apresentando, em média, $24 \%$ no verão, $25 \%$ no outono, $25 \%$ no inverno e $26 \%$ na primavera.

Muitos pesquisadores desenvolveram trabalhos sobre previsão climatológica para o Brasil, apontando a Temperatura da Superfície do Mar (TSM) como um dos principais indicadores das variações de precipitação e temperatura, sendo, portanto, um importante preditor não só para essas variáveis. Barnston \& Smith (1996) usaram a TSM para a previsão global da temperatura e precipitação pluvial, através da Análise de Correlação Canônica, em áreas afetadas pelo ENSO, como o norte e o leste da Austrália, partes da África, América do Norte e Sul, onde observaram uma habilidade satisfatória de previsão do modelo para ambas as variáveis.

As relações entre anomalias de precipitação pluvial e a TSM no Uruguai e Sul do Brasil foram investigadas por Diaz et al. (1998), nessa pesquisa pôde observar-se que a variabilidade dessa precipitação no local de estudo está ligada a anomalias de TSM dos Oceanos Pacífico e Atlântico e os resultados confirmam a relação ENOS com anomalias de precipitação pluvial durante o final da primavera e início do verão, o final do outono e início do inverno.

A previsão da precipitação pluvial sazonal na América do Sul Subtropical foi avaliada por Montecinos et al. (2000) que empregaram a TSM do Pacífico Tropical como a variável preditora. Os resultados apontam que o estudo da sazonalidade e da previsibilidade de precipitação pluvial na área de estudo, baseado na TSM do Pacífico Tropical, tem um nível significante de previsibilidade na maioria dos casos, quando encontrado em regiões e períodos bimestrais onde existe uma significante relação entre a TSM e precipitação pluvial. Isto é altamente indicativo de que a persistência de anomalias de TSM, no Pacífico Tropical, é a fonte principal de previsibilidade de chuva sazonal, quando TSM for usada como preditor.

Pezzi et al. (2000), desenvolveram um modelo regional climático chamado SIMOC (Sistema de Modelagem dos Oceanos), adaptado para prever anomalias sazonais de precipitação pluvial. O SIMOC baseia-se em uma técnica estatística denominada Análise de Correlações Canônicas (ACC) cujos preditores do modelo são os campos de TSM em áreas sobre o Pacífico e o Atlântico Tropical, e o preditando é a precipitação pluvial sobre a Região Sul do Brasil. Observou-se que o uso dos dois oceanos simultaneamente como preditores melhorou sensivelmente a precisão das previsões, quando comparados com os casos dos oceanos individuais.

Diniz (2002) estabeleceu um modelo de previsão climatológica para as temperaturas mínima e máxima em regiões homogêneas do Rio Grande do Sul, usando como variável preditora a TSM. Em um estudo mais recente realizado por Marques (2005), também utilizando a TSM como variável preditora, desenvolveu um modelo estatístico de previsão climatológica da precipitação pluvial também para o Estado do Rio Grande do Sul. Os resultados indicaram que a TSM do Oceano Pacífico, com defasagem de dois e três meses, apresenta maior influência na precipitação pluvial da região noroeste comparada à região sudeste do Estado e que a TSM do Oceano Atlântico, com defasagem de zero e um mês, apresenta relações mais evidentes na região sudeste que no noroeste do Estado. Para estas mesmas regiões, os meses de inverno apresentam os melhores ajustes nos modelos lineares derivados da TSM.

A destreza de um modelo de previsão, seja ele de tempo ou clima, está ligado à qualidade do preditor. A TSM, nos modelos climatológicos, tem demonstrado ser um preditor eficiente nos prognósticos de precipitação pluvial (MARQUES, 2005) e nas temperaturas máxima e mínima (DINIZ, 2002) em modelos desenvolvidos para o Rio Grande do Sul.

Dentro deste contexto, este trabalho teve por objetivo desenvolver um modelo de previsão climatológica para o Numero de Dia de Chuva (NDC), em algumas estações meteorológicas da metade sul do Rio Grande do Sul, usando 
como variáveis preditoras as Temperaturas da Superfície do Mar (TSM), de forma que possam prever, com antecedência de até três meses, o NDC para essas estações, estabelecendo as equações preditivas, e assim obter os valores previstos e fazer testes de eficiência do método.

\section{DADOS E METODOLOGIA}

\subsection{Dados}

Para esta pesquisa foram usados dois conjuntos de dados. O primeiro refere-se aos dados mensais de Número de Dias de Chuva (NDC) de 5 estações meteorológicas do sul do Rio Grande do Sul, para o período de 1982 a 2005, perfazendo um total de 24 anos de dados. A série foi dividida em dois períodos: o dependente, compreendendo o intervalo de 1982 a 2002, para determinação das equações preditivas, bem como dos coeficientes de regressão, e o período independente, cujo intervalo foi de 2003 a 2005, para validação do modelo. Estes dados foram obtidos junto ao Instituto Nacional de Meteorologia - INMET ( $8^{0}$ Distrito de Meteorologia - Porto Alegre), com séries já preenchidas as falhas através de técnicas apropriadas. A Tab. 1 relaciona as regiões ecoclimáticas utilizadas no trabalho e suas estações meteorológicas.

Tabela 1 - Regiões ecoclimáticas e as estações meteorológicas utilizadas.

\begin{tabular}{|l|l|}
\hline REGIÃO & ESTAÇÕES METEREOLÓGICAS \\
\hline R9 & Bagé, Uruguaina. \\
\hline R11 & $\begin{array}{l}\text { Pelotas, Rio Grande do Sul, Santa Vitória do } \\
\text { palmar. }\end{array}$ \\
\hline
\end{tabular}

O segundo conjunto de dados refere-se à Temperatura da Superfície do Mar (TSM) dos Oceanos Atlântico e Pacífico a partir de $10^{\circ} \mathrm{N}$ a $60^{\circ} \mathrm{S}$ e $120^{\circ} \mathrm{E}$ a $30^{\circ} \mathrm{E}$, numa grade de $5^{\circ} \times 5^{\circ}$, obtidos da NOAA-CIRES Climate Diagnostic Center no período de 1982 a 2005, para os meses de maio e novembro. Existem dados de TSM anterior ao ano de 1982, mas estes eram medidos espaçadamente, existiam poucos pontos de medições tipo bóias, navios e outras formas de coleta de dados. A partir de 1982, começou-se a obter mais pontos de medições nos oceanos, devido ao maior estudo direcionado ao fenômeno El Niño Oscilação Sul (resultado do forte evento 1982-1983). Com isso, houve uma concentração maior na coleta, bem como na qualidade dos dados oceânicos. E também disponibilizaram dados numa malha de distribuição espacial, que para a pesquisa, se adaptava mais ao estado do Rio Grande do Sul. A Fig. 1 mostra as áreas dos Oceanos Atlântico e Pacífico, onde foram obtidos os valores de TSM utilizados no trabalho.

\subsection{Metodologia}

Os dados de NDC das estações meteorológicas foram separados mensalmente e alocados em suas regiões ecoclimáticas (regiões de mesmas características climáticas). O total mínimo diário para considerar ocorrência de chuva foi $0,1 \mathrm{~mm}$, que de acordo com o INMET, qualquer quantidade de chuva é registrada como dia de chuva.

O modelo preditivo foi desenvolvido utilizando-se regressão linear múltipla, as séries dos dados preditantes (variáveis dependentes) e preditoras (variáveis independentes) foram obtidas da seguinte maneira: separaram-se as estações meteorológicas em suas regiões ecoclimáticas, selecionando-se as regiões ecoclimáticas que representam o sul do Rio Grande do Sul e as estações cuja série de dados de NDC apresentavamse completas ou com poucos meses de falhas preenchidas no período de estudo. Nem todas as estações meteorológicas pertencentes a uma mesma região ecoclimática foram utilizadas, então escolheram-se aquelas estações que apresentaram alto coeficiente de correlação entre seus dados. Depois de escolhidas as estações, calculou-se a média das séries de NDC para cada região, obtendo-se, dessa forma, para as regiões ecoclimáticas 9 e 11, uma série temporal média de NDC que serviram como dados preditantes para o modelo de regressão linear múltipla.

Os dados de TSM foram empregados como variáveis independentes para estabelecer os coeficientes da equação de



Figura 1 - Posições dos Oceanos Atlântico e Pacífico cujos valores da TSM foram utilizados. Fonte: Marques (2005). 
regressão. O período de defasagem é de 4 meses - tem-se um mês para o trabalho com os dados obtidos de TSM e os próximos três meses utilizados para a previsão das variáveis dependentes. As relações foram feitas entre a TSM dos meses de maio e novembro, para determinar equações preditivas do NDC para os meses de jul-ago-set e jan-fev-mar; respectivamente, como pode ser visualizado no esquema abaixo:

TSMmai $\Rightarrow$ prevendo NDC de jul-ago-set

TSMnov $\Rightarrow$ prevendo NDC de jan-fev-mar

O modelo de regressão foi construído sobre a base de dados dependentes, obtendo-se, assim, as equações de regressão, bem como seus coeficientes. A partir de então, passaram a ser usadas para prever o NDC, tanto do período dependente quanto do independente. Os coeficientes de regressão são encontrados através do uso da Técnica dos Mínimos Quadrados. Abaixo está apresentada a matriz utilizada no trabalho:
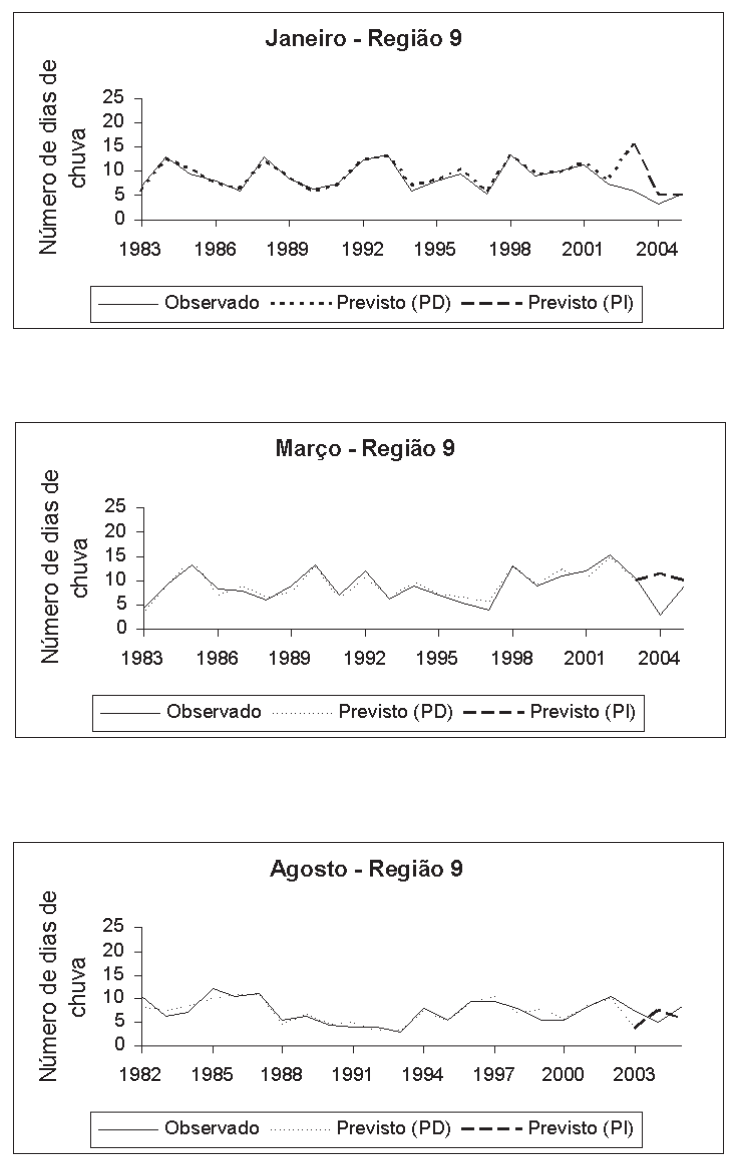

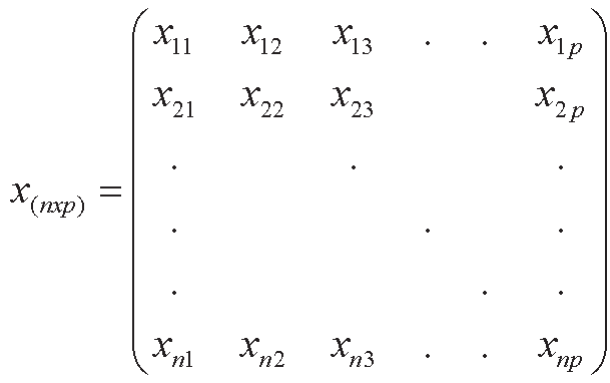

Onde:

n=24 (número de anos da série de dados, 1982-2005).

$\mathrm{p}=675$ (totais de pontos com séries temporais mensais de Número de Dias de Chuva e séries temporais de TSM).

Depois de estabelecidas as equações, foram calculados os valores de NDC, para serem comparados com os observados, a fim de avaliar o modelo. No processo de validação do modelo, utilizou-se o erro relativo $(\varepsilon)$ que permite avaliar se o método nos fornece resultados aceitáveis ou se estes não são adequados.
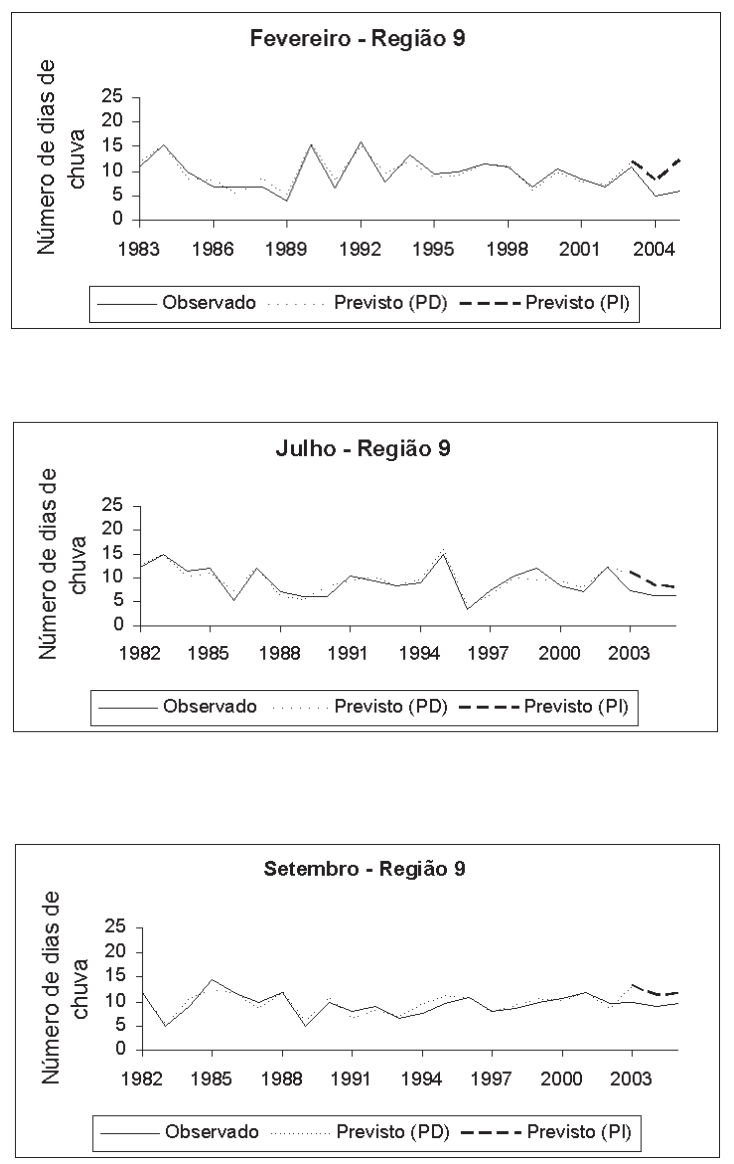

Figura 2 - Série do Número de Dias de Chuva previsto e observado para os períodos dependente (PD), 1982-2002 e independente (PI), 2003-2005 para a Região 9. 
O erro relativo foi calculado tanto para o período dependente como para o período independente, com a finalidade de analisar, separadamente, a eficácia do modelo. A correlação foi calculada entre os dados observados e previstos, para considerar a relação entre eles, bem como o skill (precisão) do método. Também para avaliar o desempenho do método foi calculado o desvio médio quadrático (DMQ), este é frequentemente usado para medir as diferenças entre os valores previstos e observados.

Para melhor relacionar os dados observados com os dados previstos de NDC são também apresentadas as estatísticas do NDC, como as médias mensais, o desvio padrão, e o coeficiente de variação do período estudado.

\section{RESULTADOS}

\subsection{Relação entre dados previstos e observados do Número de Dias de Chuva em estações meteorológicas das regiões ecoclimáticas}
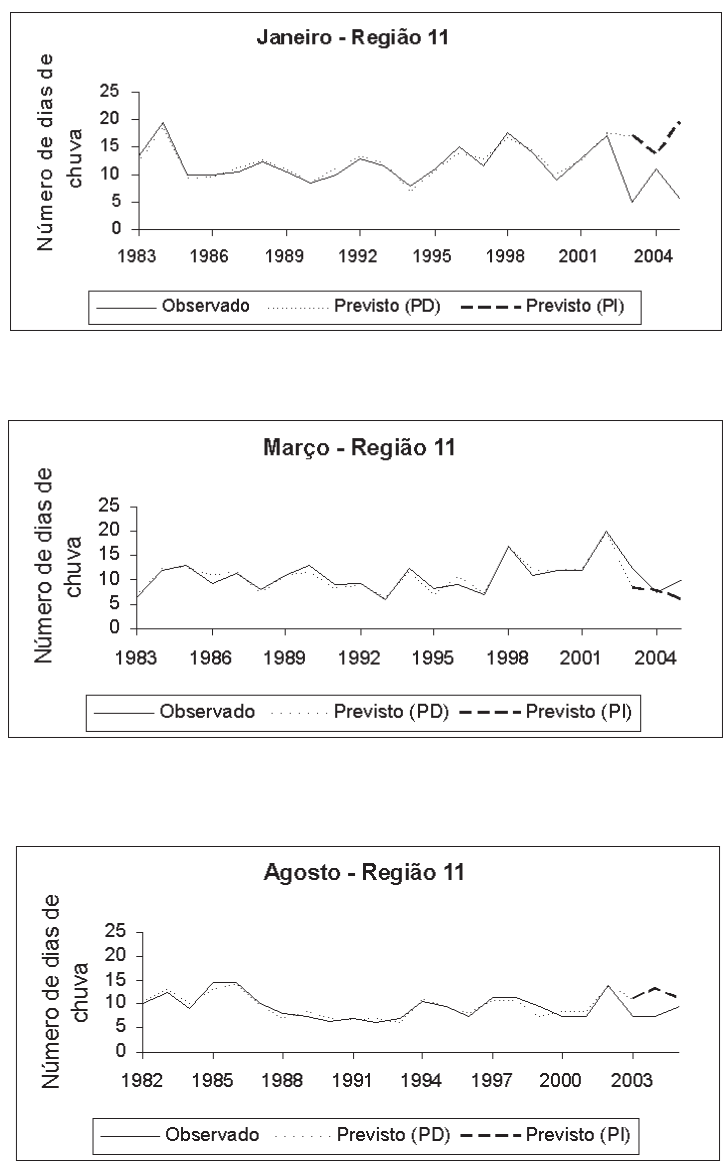

Conforme descrito na metodologia, a partir da série de dados observados, foi construída a série de dados prevista, utilizando os últimos 3 anos da série (2003 a 2005) como período independente para a validação do método. A apresentação dos resultados foi feita de forma a comparar as séries de dados, analisando-se cada região ecoclimática separadamente, a fim de encontrar qual delas teve o melhor resultado. A seguir são mostrados os resultados das séries previstas e as observadas das duas regiões ecoclimáticas analisadas. Os gráficos do mês de janeiro apresentam dados a partir de 1983 porque, para prever os três primeiros meses do ano de 1982, seriam necessários dados de TSM de 1981, e neste trabalho foram empregados dados de TSM a partir do ano de 1982.

A Fig. 2 mostra os resultados das séries calculadas e observadas para os três meses de verão e três meses de inverno, referente à região ecoclimática $\mathrm{R} 9$.

A região ecoclimática $\mathrm{R} 9$ obteve excelentes resultados em todos os meses observados. As séries de dados previstas
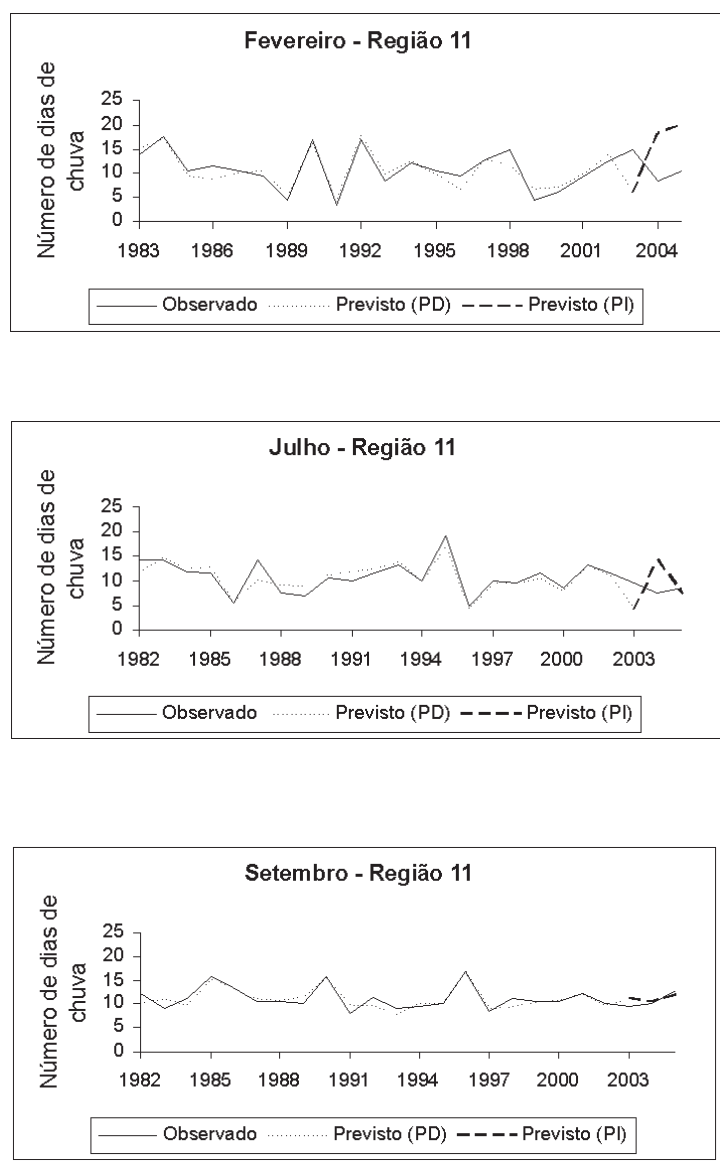

Figura 3 - Série do Número de Dias de Chuva previsto e observado para os períodos dependente (PD), 1982-2002 e independente (PI), 2003-2005 para a Região 11. 
e observadas seguem um mesmo padrão de distribuição da variável estudada; existem alguns picos em que os valores previstos apresentam diferenças dos valores observados, mas não são diferenças significativas. O período dependente apresentou bons resultados para os todos os meses analisados, já o período independente apresentou melhores resultados no trimestre de inverno (jul-ago-set).

A Fig. 3 mostra os resultados das séries calculadas e observadas para os três meses de verão e três meses de inverno, referente à região ecoclimática R11.

Nesta região, para o período dependente, o modelo mostra bons resultados para todos os meses observados, no entanto o período independente apresenta maiores diferenças entre valores observados e previstos, podendose ressaltar o mês de janeiro, onde estas diferenças ficam mais visíveis. Pode-se destacar o trimestre do inverno por apresentar resultados melhores que o trimestre do verão.

Os resultados de Marques (2005) indicaram que os meses de inverno apresentam os melhores ajustes nos modelos lineares derivados da TSM, na previsão da precipitação pluvial do Rio Grande do Sul.

Os oceanos, diferente dos continentes, levam algum tempo para responder ao aquecimento e resfriamento, em função de ser um termoregulador no sistema oceano-atmosfera. Devido a essas variações, as TSM de alguns meses, principalmente as do outono, são melhores preditores do que outros meses.

\subsection{Estatísticas do Número de Dias de Chuva Observado e Previsto, das estações meteorológicas utilizadas}

Os parâmetros estatísticos do NDC para as séries observadas e previstas, tais como média, desvio padrão e coeficiente de variação das estações meteorológicas utilizadas na pesquisa são apresentadas nas Tabs. 2 e 3. Pode-se verificar que existem pequenas diferenças entre as médias de NDC observadas e previstas, estas diferenças não chegam a ser de um dia de chuva.

Tabela 2 - Parâmetros estatísticos do Número de Dias de Chuva Observado; Média, Desvio Padrão e Coeficiente de Variação das estações meteorológicas utilizadas na pesquisa, Rio Grande do Sul, 1982 - 2005.

\begin{tabular}{lllllll}
\multicolumn{7}{c}{ MED. } \\
\hline Região/mês & Jan & Fev & Mar & Jul & Ago & Set \\
R9 & 8.7 & 9.5 & 9.0 & 9.3 & 7.4 & 9.5 \\
R11 & 11.6 & 10.9 & 10.8 & 10.6 & 9.4 & 11.1 \\
\hline & & & D.P. & & & \\
\hline R9 & 2.90 & 3.36 & 3.31 & 3.09 & 2.53 & 2.20 \\
R11 & 3.52 & 3.99 & 3.26 & 3.12 & 2.54 & 2.38 \\
\hline & & & C.V. & & & \\
\hline R9 & 33.4 & 35.5 & 36.9 & 33.4 & 34.5 & 23.3 \\
R11 & 30.3 & 36.7 & $\mathbf{3 0 . 2}$ & 29.5 & 27.0 & 21.3 \\
\hline
\end{tabular}

Tabela 3 - Parâmetros estatísticos do NDC Previsto; Média, Desvio Padrão e Coeficiente de Variação das estações meteorológicas utilizadas na pesquisa, Rio Grande do Sul, 1982 - 2005.

MED.

\begin{tabular}{lllllll}
\hline Região/mês & Jan & Fev & Mar & Jul & Ago & Set \\
R9 & 9.1 & 9.9 & 9.4 & 9.5 & 7.2 & 9.8 \\
R11 & 12.9 & 11.3 & 10.4 & 10.6 & 9.9 & 11.2 \\
\hline & \multicolumn{7}{c}{ D.P. } & & & \\
\hline R9 & 3.07 & 3.04 & 2.91 & 2.81 & 2.41 & 2.15 \\
R11 & 3.29 & 4.49 & 3.26 & 3.21 & 2.47 & 2.10 \\
\hline & & & C.V. & & & \\
\hline R9 & 33.5 & 30.7 & 31.1 & 29.5 & 33.5 & 22.0 \\
R11 & 25.6 & 39.6 & 31.2 & 30.3 & 25.0 & 18.7 \\
\hline
\end{tabular}

Analisando o desvio padrão da série de NDC observada nota-se que este varia de 2,20 a 3,99, já para a série prevista o desvio padrão varia de 2,10 a 4,49, comparando as regiões R9 e R11.

Quanto ao coeficiente de variação para a série observada varia de $21,35 \%$ a $36,9 \%$, para a série prevista varia de $18,7 \%$ a $39,6 \%$.

Nota-se que, tanto para os valores do desvio padrão como para os valores do coeficiente de variação, o trimestre de inverno possui os menores valores destes parâmetros estatísticos, sendo o mês de setembro o de melhor resultado.

\subsection{Avaliação do desempenho do método através do Coeficiente de Correlação entre a série de dados observada e a série de dados prevista}

O coeficiente de correlação (r) entre a série de dados observada e a série de dados prevista são mostradas na Tab. 4 , onde se pode observar que apenas um coeficiente registrou valor menor que 0,50. A região R9 apresentou os maiores coeficientes de correlação, caracterizando a região de melhor skill, e a região R11 obteve coeficientes de valores um pouco menores. Os meses de março, julho e setembro obtiveram os maiores coeficientes de correlação e o mês de janeiro obteve os valores mais baixos.

Conforme valores calculados, levando em consideração o número de pares da amostra, qualquer coeficiente de correlação da Tab. 4 maiores que 0,47 são significativos a $1 \%$, os demais são significativos a $5 \%$.

\subsection{Avaliação do desempenho do método através do Desvio Médio Quadrático}

A Tab. 5 apresenta os valores de DMQ, para todo o período em estudo. Os valores variam de 0,06 a 6,05 . Sendo o mês de julho para R11 que apresentou o menor valor de DMQ e de uma maneira geral, o trimestre de inverno obteve os melhores resultados. 
Tabela 4 - Coeficientes de correlação entre a série de dados observada e prevista. Período, 1982 - 2005.

\begin{tabular}{lcc}
\hline Mês & Região 9 & Região 11 \\
\hline Janeiro & 0,74 & 0,37 \\
Fevereiro & 0,85 & 0,62 \\
Março & 0,81 & 0,91 \\
Julho & 0,90 & 0,76 \\
Agosto & 0,84 & 0,79 \\
Setembro & 0,83 & 0,89 \\
\hline
\end{tabular}

Tabela 5 - Desvio médio quadrático (DMQ) para Número de Dias de Chuva. Período, 1982 - 2005.

\begin{tabular}{lcc}
\hline Mês & Região 9 & Região 11 \\
\hline Janeiro & 2.17 & 6.05 \\
Fevereiro & 2.06 & 2.21 \\
Março & 1.86 & 1.61 \\
Julho & 1.43 & 0.06 \\
Agosto & 0.78 & 2.27 \\
Setembro & 1.53 & 0.20 \\
\hline
\end{tabular}

Para o período independente Tab. 6 os valores de DMQ encontram-se muito próximos de zero, caracterizando um excelente resultado. Já para o período independente Tab. 7 o DMQ apresentou valores um pouco maiores do que para o período dependente, variando de 0 a 16,45, mesmo assim, obteve-se bons resultados.

Observando as Tabs. 6 e 7 nota-se também que o trimestre de inverno obteve os melhores valores.

Tabela 6 - Desvio médio quadrático (DMQ) para Número de Dias de Chuva. Período, 1982 - 2002.

\begin{tabular}{lcc}
\hline Mês & Região 9 & Região 11 \\
\hline Janeiro & 0.07 & 0.11 \\
Fevereiro & 0.09 & 0.00 \\
Março & 0.04 & 0.04 \\
Julho & 0.07 & 0.07 \\
Agosto & 0.04 & 0.00 \\
Setembro & 0.04 & 0.02 \\
\hline
\end{tabular}

Tabela 7 - Desvio médio quadrático (DMQ) para Número de Dias de Chuva. Período, 2003 - 2005.

\begin{tabular}{lcc}
\hline Mês & Região 9 & Região 11 \\
\hline Janeiro & 6.18 & 16.45 \\
Fevereiro & 5.95 & 6.12 \\
Março & 5.02 & 4.56 \\
Julho & 3.87 & 0.00 \\
Agosto & 2.31 & 6.41 \\
Setembro & 4.21 & 0.52 \\
\hline
\end{tabular}

\subsection{Variância das equações prognósticas de Número de Dias de Chuva}

A análise das equações foi feita através da variância explicada pelas variáveis independentes (TSM) em relação à variável dependente (Número de Dias de Chuva). As Tabelas 8 e 9 mostram as proporções da variância explicada das equações prognósticas, cujas variáveis dependentes são de um mês e, as independentes, dos meses anteriores (defasagem 1, 2, 3, 4), como por exemplo na Tab. 9: defasagem de 1 mês, (trabalho com os dados para a elaboração do prognóstico); defasagem de 2 meses - maio prevendo julho; defasagem de 3 meses maio prevendo agosto; defasagem de 4 meses - maio prevendo setembro.

Verifica-se que as variâncias explicadas dos modelos prognósticos obtiveram ótimos resultados, nenhum valor ficou abaixo de $70 \%$. O mês de novembro, utilizado como preditor dos meses de janeiro, fevereiro e março, apresentou valores de variância ligeiramente maiores que o mês de maio. Com os presentes resultados, para novembro como preditor, a região R11 e R9 apresentam altos valores de variância explicada, com uma média de 91,5 e 91,3 respectivamente. Com isso pode-se dizer que o mês de novembro é um bom preditor.

Tabela 8 - Variância explicativa (\%) das equações prognósticas, de Número de Dias de Chuva para julho, agosto e setembro com a TSM de maio, período de 1982 a 2002.

\begin{tabular}{ccc}
\hline Mês & Região 9 & Região 11 \\
\hline Mai./jul. & 87,8 & 82,0 \\
Mai./ago. & 85,7 & 88,2 \\
Mai./set. & 80,3 & 81,0 \\
\hline
\end{tabular}


Tabela 9 - Variância explicativa (\%) das equações prognósticas, de Número de Dias de Chuva para janeiro, fevereiro e março com a TSM de novembro, período de 1982 a 2002.

\begin{tabular}{ccc}
\hline Mês & Região 9 & Região 11 \\
\hline Nov./jan. & 94,8 & 93,0 \\
Nov./fev. & 89,0 & 87,5 \\
Nov./mar & 90,0 & 94,1 \\
\hline
\end{tabular}

\subsection{Teste do modelo}

Uma das formas de aferição quanto à eficiência do modelo adotado neste trabalho foi realizada através do erro relativo (e). Essa metodologia foi também usada por Diniz \& Calvetti (1998), Gonçalves \& Kim (1998) e Puchalski (2000). O método foi aplicado primeiramente para o período dependente e num segundo momento para o período independente. As Tabelas 10 e 11 listam o erro relativo da previsão do NDC para as duas regiões ecoclimáticas utilizadas, para o período dependente e independente, respectivamente.

Verifica-se na Tab. 10 que os erros relativos das duas regiões estão abaixo de 1 , o que caracteriza a eficiência do modelo. A região que obteve os erros relativos mais baixos foi a região $\mathrm{R} 9$, com um erro relativo médio de 0,31 . Os meses que apresentaram os menores erros relativos foram janeiro e março, com média de 0,25 , já setembro obteve os maiores valores de erros relativos, com media de 0,4 . Com isso, pode-se dizer que houve excelentes resultados para o período dependente. Estes resultados estão relacionados com a variância explicativa dos meses citados, pois os meses com menores erros relativos possuem maiores valores de variância explicada.

Tabela 10 - Erro relativo (e) da previsão do Número de Dias de Chuva para as regiões ecoclimáticas, período de 1982 a 2002.

\begin{tabular}{ccc}
\hline Mês & Região 9 & Região 11 \\
\hline Nov./jan. & 94,8 & 93,0 \\
Nov./fev. & 89,0 & 87,5 \\
Nov./mar & 90,0 & 94,1 \\
\hline
\end{tabular}

Na Tabela 11, somente dois valores de erros relativos encontram valores abaixo de 1 . A região que obteve os melhores resultados foi a $\mathrm{R} 9$, com um erro relativo médio de 1,31 , sendo setembro o mês de melhor resultado, com média 0,8 . O mês de janeiro registrou os maiores valores de erros relativos.
Tabela 11 - Erro relativo (e) da previsão do Número de Dias de Chuva para as regiões ecoclimáticas, período de 2003 a 2005.

\begin{tabular}{lcc}
\hline Mês & Região 9 & Região 11 \\
\hline Janeiro & 2,2 & 3,6 \\
Fevereiro & 1,2 & 2,3 \\
Março & 1,5 & 1,0 \\
Julho & 0,8 & 1,6 \\
Agosto & 1,1 & 1,6 \\
Setembro & 1,1 & 0,5 \\
\hline
\end{tabular}

Um dos fatos do erro relativo apresentar valores bem mais altos no período independente é que, dependendo do período utilizado no desenvolvimento do modelo, as condições oceânicas não apresentam uma variedade maior de situações anômalas e estas situações acontecerem no período de validação do modelo (período independente), criando uma situação nova que não entrou no cálculo dos coeficientes de regressão das equações preditivas.

Deve-se ressaltar que esta pesquisa, por se tratar de previsão climática utilizando modelos estatísticos, referiu-se principalmente ao enfoque estatístico puro e simples, sem se aprofundar nas questões envolvendo processos físicos e dinâmicos da interação Oceano-Atmosfera.

\section{CONCLUSÃO}

A partir da metodologia proposta, os resultados obtidos permitem concluir que:

A Temperatura da Superfície do Mar (TSM) é um bom preditor para o Número de Dias de Chuva (NDC). A defasagem temporal ideal entre essas variáveis no desenvolvimento de modelos preditivos é de até quatro meses.

Para as duas regiões analisadas, obtiveram-se bons resultados na previsão de NDC. A série de dados prevista e a série de dados observada seguem um mesmo padrão de distribuição desta variável, entretanto, existem alguns valores previstos que apresentam diferenças dos valores observados, mas não são diferenças significativas. No período independente, a série prevista mostra as maiores diferenças em relação aos valores observados.

O modelo apresentou uma maior habilidade na previsão de NDC para o trimestre de inverno do que para o trimestre de verão.

\section{REFERÊNCIAS BIBLIOGRÀFICAS}

BARNSTON, A.G.; SMITH, T.M. Specification and prediction of global surface temperature and precipitation from global SST using CCA. Journal of Climate, v.9, n. 11, p. 26602697, 1996. 
BERLATO, M.A. As condições de precipitação pluvial no Estado do Rio Grande do Sul e os impactos das estiagens na produção agrícola. In: BERGAMASCHI, H. (Coord.) Agrometeorologia aplicada à irrigação. Porto Alegre: Editora da Universidade/UFRGS, p. 11-23, 1992.

DIAZ, A.F.; STUDZINSKI, C.D.; MECHOSO, C.R. Relationships between precipitation anomalies in Uruguay and Southern Brazil and sea surface temperature in the Pacific and Atlantic oceans. Journal of Climate, Boston, v. 11, n. 2, p. 251-271, 1998.

DINIZ, G.B. Determinação de preditores informativos visando um modelo de previsão climática de temperatura máxima e mínima para regiões homogêneas do Rio Grande do Sul. 2002. 167 f. Tese (Doutorado - Agrometeorologia) - Programa de Pós-Graduação em Fitotecnia, Faculdade de Agronomia, Universidade Federal do Rio Grande do Sul, Porto Alegre, 2002.

DINIZ, G.B.; CALVETTI, L. Regiões informativas de TSM (Hemisfério Sul) para previsão de temperatura máxima em algumas estações do Rio Grande do Sul. In: CONGRESSO BRASILERIO DE METEOROLOGIA, 10.; CONGRESSO DA FLISMET, 8., 1998, Brasília. Anais... Rio de Janeiro: SBMet, 1998. CL-98137. 1 CD-ROM.

FONTANA, D.C; ALMEIDA, T.S. Climatologia do número de dias de chuva com precipitação pluvial no Estado do Rio Grande do Sul. Revista Brasileira de Agrometeorologia,
Santa Maria -RS, v.10, n.1, p.135-145, 2002.

GONÇALVES, F.V.; KIM, I.S. Recuperação de dados climatológicos do Rio Grande do Sul e Santa Catarina. In: CONGRESSO BRASILERIO DE METEOROLOGIA, 10.; CONGRESSO DA FLISMET, 8.; 1998, Brasília. Anais... Rio de Janeiro: SBMet, 1998. Cl-98173. 1 CD-ROM.

MARQUES, J. R. Variabilidade espacial e temporal de precipitação pluvial no Rio Grande do Sul e sua relação com indicadores oceânicos. 2005. 210f. Tese (DoutoradoAgrometeorologia) - Programa de Pós-graduação em Fitotecnia, Faculdade de Agronomia, Universidade Federal do Rio Grande do Sul, Porto Alegre, 2005.

MONTECINOS, A.; DÍAZ, A.; ACEITUNO, P. Seasonal diagnostic and predictability of rainfall in Subtropical South America based on Tropical Pacific SST. Journal of Climate, v. 13, n. 4, p. 746-758, 2000.

PEZZI, L.P.; UBARANA, V.; REPELLI, C. Desempenho e previsão de um modelo regional estatístico para a Região Sul do Brasil. Revista Brasileira de Geofísica, v. 18, n.2, p. 129-146, 2000.

PUCHALSKI, L. A. Efeito associado ao El niño e La Niña na temperatura média, precipitação pluvial e no déficit hídrico no Rio Grande do Sul. 200. 100 f. Dissertação (Mestrado - Agrometeorologia) - Programa de Pós-graduação em Fitotecnia, Faculdade de Agronomia, Universidade Federal do Rio Grande do Sul, Porto Alegre, 2000. 